\title{
A re-evaluation of the taxonomy of the genus Anaerovibrio, with the reclassification of Anaerovibrio glycerini as Anaerosinus glycerini gen. nov., comb. nov., and Anaerovibrio burkinabensis as Anaeroarcus burkinensis [corrig.] gen. nov., comb. nov.
}

\author{
Carsten Strömpl, ${ }^{1}$ B. J. Tindall, ${ }^{3}$ Graeme N. Jarvis, ${ }^{4} \dagger$ Heinrich Lünsdorf, ${ }^{2}$ \\ Edward R. B. Moore ${ }^{1}$ and Hans Hippe ${ }^{3}$
}

\author{
Author for correspondence: Carsten Strömpl. Tel: +49531 6181 405. Fax: +495316181411. \\ e-mail: cst@gbf.de
}

\footnotetext{
1.2 Microbial Ecology Group 1 and Electron Microscopy Unit2, Division of Microbiology, Gesellschaft für Biotechnologische Forschung $\mathrm{mbH}$, Mascheroder Weg 1, D-38124 Braunschweig, Germany

3 Deutsche Sammlung von Mikroorganismen und Zellkulturen, Mascheroder Weg 1b, D38124 Braunschweig. Germany

${ }^{4}$ Rumen Microbiology Unit, AgResearch Grasslands Centre, Private Bag 11008, Palmerston North, New Zealand
}

\begin{abstract}
Chemotaxonomic, electron microscopic and 16S rRNA gene sequence analyses of the three described species of the genus Anaerovibrio demonstrated only remote similarities to each other. The 165 rRNA gene sequence similarities between Anaerovibrio lipolytica, Anaerovibrio glycerini and Anaerovibrio burkinabensis and the derived phylogenetic relationships of the three species studied fell below genus level. All three species clustered within the Sporomusa-Pectinatus-Selenomonas phyletic group. Each species showed a distinct phospholipid pattern and whole-cell fatty acid distribution. Several isoprenologues of the lipoquinone 'lipid F' were found to differ in their quantitative distribution in the Anaerovibrio species. On the basis of these results, the new genera Anaerosinus gen. nov. and Anaeroarcus gen. nov. are proposed. The type species of Anaerosinus is Anaerosinus glycerini comb. nov. and the type species of Anaeroarcus is Anaeroarcus burkinensis [corrig.] comb. nov. The genus Anaerovibrio is consequently restricted to a single species, namely Anaerovibrio lipolyticus [corrig.].
\end{abstract}

Keywords: Anaerovibrio, Sporomusa-Pectinatus-Selenomonas phyletic group, polyphasic taxonomy, Anaerosinus gen. nov., Anaeroarcus gen. nov.

\section{INTRODUCTION}

Hungate (1966) created the genus Anaerovibrio to accommodate a vibrioid, Gram-negative, obligately anaerobic, lipolytic bacterium that had been isolated from the rumen of sheep (Hobson \& Mann, 1961). The genus included a single species, Anaerovibrio lipolytica,

\footnotetext{
tPresent address: Section of Microbiology, 156 Wing Hall, Cornell University, Ithaca, NY 14853, USA.

Abbreviation : SSCP, single-strand conformation polymorphism.

The GenBank/EMBLDDBJ accession numbers for the 165 rRNA gene sequences determined in this study are as follows: AJ010959 (Anaerovibrio lipolytica DSM 3074'), AJ010960 (Anaerovibrio glycerini DSM 5192'), AJ010961 (Anaerovibrio burkinabensis DSM 6283'), AJ010962 (Clostridium quercicolum DSM 1736'), AJ010963 (Centipeda periodontii DSM 2778') and AJ010964 (Acetonema longum DSM 6540').
}

and was later assigned to the family Bacteroidaceae, together with other anaerobic, Gram-negative organisms (Prins, 1986). Two additional species have been assigned to the genus. Anaerovibrio glycerini was isolated from anoxic freshwater sediments (Schauder \& Schink, 1989), and Anaerovibrio burkinabensis was isolated from rice-field soils (Ouattara et al., 1992). The type species, Anaerovibrio lipolytica, was believed to occupy both the lactate-utilizing and lipid-degrading ecological niches within the rumen ecosystem (Hungate, 1966) and was observed to utilize only a limited number of saccharides and glycerol as growth substrates. Anaerovibrio glycerini is capable of growth on only two substrates, glycerol and the glycerol portion of diolein (Schauder \& Schink, 1989). It is unable to utilize lactate or hydrolyse lipids and lipid analogues (Schauder \& Schink, 1989). In contrast, the 
third member of the genus, Anaerovibrio burkinabensis, grows on a wide range of saccharide substrates, is capable of lactate utilization, but exhibits no lipolytic activity (Ouattara et al., 1992). Apart from the differences in the metabolic traits of the three Anaerovibrio species, the wide range of $\mathrm{G}+\mathrm{C}$ contents of Anaerovibrio lipolytica, Anaerovibrio glycerini and Anaerovibrio burkinabensis $(31 \cdot 5,34 \cdot 3$ and $44 \cdot 1 \mathrm{~mol} \%$ respectively) also indicates that the genus is genetically diverse.

The introduction of 16S rRNA gene sequence-based phylogenetic analyses into bacterial taxonomy during the last two decades (see Woese, 1987) has led to new insights into the evolutionary relationships of prokaryotes. Chemotaxonomic studies have also made a valuable contribution to the delineation of taxa that are otherwise difficult to characterize. In the present study, the 16S rRNA gene sequences and lipid composition of the three species described, comprising the genus Anaerovibrio, are reported. On the basis of these results, a reclassification of two of the species into two new genera is proposed.

\section{METHODS}

Organisms and growth conditions. The type strains of Anaerovibrio lipolytica (DSM 3074 $)$, Anaerovibrio glycerini (DSM 5192 $2^{\mathrm{T}}$ ) and Anaerovibrio burkinabensis (DSM 6283 were obtained from the German Collection of Microorganisms and Cell Cultures (DSMZ). All strains were grown anaerobically in peptone/yeast extract (PY) broth (Holdeman et al., 1977) supplemented with $0.5 \%(\mathrm{v} / \mathrm{v})$ glycerol (Anaerovibrio lipolytica, Anaerovibrio glycerini) or $0.25 \%(\mathrm{v} / \mathrm{v})$ lactate (Anaerovibrio burkinabensis). Growth temperatures were $30^{\circ} \mathrm{C}$ (Anaerovibrio glycerini), $35^{\circ} \mathrm{C}$ (Anaerovibrio burkinabensis) and $37^{\circ} \mathrm{C}$ (Anaerovibrio lipolytica). The culture head space contained $\mathrm{N}_{2} / \mathrm{CO}_{2}, 1: 1(\mathrm{v} / \mathrm{v})$ in Hungate tubes and a $4: 1(\mathrm{v} / \mathrm{v})$ ratio in 4.51 cultures. To enable comparison with existing fatty acid data, all strains were also grown in peptone/yeast extract/glucose (PYG) broth (Holdeman et al., 1977; Moore et al., 1994).

Electron microscopy. Anaerovibrio glycerini cells from PY/ glycerol broth were harvested in the mid-exponential phase of growth and used for electron microscopic studies. Cells were fixed in $2.5 \%(\mathrm{v} / \mathrm{v})$ glutardialdehyde, either in the growth medium or in PBS ( $\mathrm{pH} 7 \cdot 2$ ), for ultrathin-section preparations. The cells were then immobilized with $2 \%$ (w/v) Noble agar (Difco), washed with PBS buffer and then in $0.1 \mathrm{M}$ cacodylate buffer ( $\mathrm{pH} \mathrm{7.2)}$; the cells were then fixed in cacodylate buffer solution $(0 \cdot 1 \mathrm{M}, \mathrm{pH} 7 \cdot 2)$ containing $1 \%$ $(\mathrm{w} / \mathrm{v}) \mathrm{OsO}_{4}$. Dehydration through an acetone series and resin embedding were carried out using the procedures of Spurr (1969). Ultrathin sections $(100-120 \mathrm{~nm})$ were cut using a Reichert Ultracut S (Leica) ultramicrotome, picked up with Formvar-coated grids $(\mathrm{Cu}, 300$ mesh) and poststained with aqueous uranyl acetate and lead citrate using the procedures of Reynolds (1963). Samples were observed using a Zeiss CEM 902 transmission electron microscope at primary magnifications of $\times 7000-12000$.

DNA isolation and base composition. Genomic DNA was isolated and purified using Qiagen Genomic Tips (Qiagen), according to the manufacturer's instructions, from $2 \mathrm{ml}$ samples of liquid cultures grown in the media described above. Appropiate amounts of DNA were enzymically digested and the mean $\mathrm{G}+\mathrm{C}$ contents were determined using HPLC (Tamaoka \& Komagata, 1984). Calculations of nucleoside ratios were carried out according to Mesbah et al. (1989), with non-methylated lambda-phage DNA (Sigma/ Aldrich) as a standard.

16S rDNA sequencing. Nearly complete 16S rRNA genes were amplified from chromosomal DNA by using the PCR (Mullis \& Faloona, 1987) using a forward primer hybridizing at positions 8-27 (Escherichia coli rRNA gene numbering, primer sequence 5'-GAGTTTGATCMTGGCTCAG-3') and a reverse primer hybridizing at the complement of positions 1492-1512 (5'-CGGTTACCTTGTTACGGACTT $\left.-3^{\prime}\right)$. The PCR was carried out using a GeneAmp 9600 thermocycler (Perkin-Elmer) and conditions described previously (Karlson et al., 1993). Amplified DNA was purified with Microcon 100 microconcentrators (Amicon) and the quality was checked using gel electrophoresis on a $1 \%(\mathrm{w} / \mathrm{v})$ agarose gel with Tris-acetate/EDTA (TAE) buffer and subsequent ethidium bromide staining. The sequences of the amplified 16S rRNA gene were determined with an Applied Biosystems 373A DNA sequencer using the protocols recommended by the manufacturer for Taq-polymeraseinitiated cycle sequencing with fluorescent-dye-labelled dideoxynucleotides and standard 16S rRNA sequencing primers (Lane, 1991). The resulting sequences were aligned with reference 16S rRNA and 16S rRNA gene sequences (Maidak et al., 1999; Stoesser et al., 1999; van de Peer et al., 1996) using evolutionarily conserved primary sequence and secondary structure (Gutell et al., 1985; Neefs et al., 1991) as references. Evolutionary distances (Jukes \& Cantor, 1969) were calculated from sequence-pair similarities, using only unambiguously determined nucleotide positions. Phylogenetic trees were constructed using the DNADIST and FITCH programs of the PHYLIP package, version 3.2 (Felsenstein, 1989).

Extraction of respiratory lipoquinones and polar lipids. Respiratory lipoquinones and polar lipids were extracted from $100 \mathrm{mg}$ freeze-dried cell material using the two-stage method described by Tindall (1990a, b). Respiratory lipoquinones were extracted using methanol:hexane, and the polar lipids were extracted by adjusting the remaining methanol $/ 0 \cdot 3 \%$ aqueous $\mathrm{NaCl}$ phase (containing the cell debris) to give a chloroform $/$ methanol $/ 0 \cdot 3 \%$ aqueous $\mathrm{NaCl}$ mixture $(1: 2: 0 \cdot 8$, by vol.). The extraction solvent was stirred overnight and the cell debris pelleted by centrifugation. Polar lipids were recovered into the chloroform phase by adjusting the chloroform/methanol $/ 0 \cdot 3 \%$ aqueous $\mathrm{NaCl}$ mixture to a ratio of $1: 1: 0 \cdot 9$ (by vol.).

Analysis of respiratory lipoquinones. Respiratory lipoquinones were separated into their different classes (naphthoquinones and benzoquinones) by TLC on silica gel (Macherey-Nagel, Art. No. 805 023), using hexane/tertbutylmethylether $(9: 1, \mathrm{v} / \mathrm{v})$ as the solvent (Tindall, 1996). UV-absorbing bands corresponding to naphthoquinones or benzoquinones were removed from the plate and further analysed by HPLC. This step was carried using LDC Analytical (Thermo Separation Products) HPLC equipment fitted with a reverse-phase column (Macherey-Nagel, $2 \mathrm{~mm}$ $\times 125 \mathrm{~mm}, 3 \mu \mathrm{m}$, RP18) using methanol as the eluant. Respiratory lipoquinones were detected at a wavelength of $269 \mathrm{~nm}$.

Analysis of polar lipids. Polar lipids were separated by twodimensional, silica-gel TLC (Macherey-Nagel, Art. No. 818 135) (Stahl, 1967). The first direction was developed in 
chloroform/methanol/water $(65: 25: 4$, by vol.) and the second in chloroform/methanol/acetic acid/water $(80: 12: 15: 4$, by vol.). Total lipid material and specific functional groups were detected using dodecamolybdophosphoric acid (total lipids), Zinzadze reagent (phosphate), ninhydrin (free amino groups), periodate-Schiff reagent $(\alpha$ glycols), Dragendorff reagent (quaternary nitrogen) and anisaldehyde/sulfuric acid (glycolipids).

Fatty acid analysis. Fatty acids were analysed as the methyl ester derivatives prepared from $10 \mathrm{mg}$ dry cell material. Cells were subjected to differential hydrolysis in order to detect ester-linked and non-ester-linked (amide-bound) fatty acids (B. J. Tindall, unpublished results; Miller, 1982). Fatty acid methyl esters were analysed by GC with a $0 \cdot 2 \mu \mathrm{m} \times 25 \mathrm{~m}$ non-polar capillary column and flame-ionization detection. The run conditions were as follows: injection and detector port temperature, $300{ }^{\circ} \mathrm{C}$; inlet pressure, $60 \mathrm{kPa}$; split ratio, $50: 1$; injection volume, $1 \mu \mathrm{l}$; temperature programme, 130 $310^{\circ} \mathrm{C}$ at a rate of $4{ }^{\circ} \mathrm{C} \mathrm{min}{ }^{-1}$. Helium was used as the carrier gas.

GC-MS analysis of the fatty acids was carried out using a Finnigan MAT GCQ. The gas chromatograph was equipped with a DB- 5 column $(30 \mathrm{~m} \times 0.22 \mathrm{~mm}$; film thickness, $0.25 \mu \mathrm{m}$ ). Helium, at a linear velocity of $40 \mathrm{~cm} \mathrm{~s}^{-1}$, was used as the carrier gas. A $1 \mu$ laliquot of sample was injected in the splitless mode and separated using a temperature programme starting at $80^{\circ} \mathrm{C}$ (held for $1 \mathrm{~min}$.) followed by a ramp of $10^{\circ} \mathrm{C} \mathrm{min}^{-1}$ to $300^{\circ} \mathrm{C}$. The mass spectrometer was run in the EI mode, with a source temperature of $175^{\circ} \mathrm{C}$ and a transfer line temperature of $275^{\circ} \mathrm{C}$. Samples were run either without derivatization or as the trimethyl silane derivatives of hydroxylated fatty acids formed.

\section{RESULTS}

\section{Morphological characterization}

Anaerovibrio lipolytica and, to a lesser degree, Anaerovibrio burkinabensis, have been studied in detail by electron microscopy and their cell morphologies have been established (Henderson \& Hodgkiss, 1973; Ouattara et al., 1992). Such data were lacking for Anaerovibrio glycerini. Therefore, it was subjected to electron microscopic examination intended, in particular, to characterize its cell wall type and flagellation. Anaerovibrio glycerini possessed a typical vibrioid morphology when ultrathin sections were analysed by electron microscopy (Fig. 1). Although the sample analysed represented exponentially growing cells, the cytoplasm of individual cells showed differences in electron opacity, i.e. some of the cells appeared less electrondense than others (Fig. 1a, asterisks). In parallel with the high electron density of the cytoplasm, some of these cells showed a localized concentration of the bacterial chromosome (Fig. 1a, arrows). Septum fissure was not accompanied by formation of a narrow division cleft. A broad, conical deformation of the cell body at the septum was apparent when cell division was in progress (Fig. 1b, arrow). It was obvious from ultrastructural details (Fig. 1c) that the cell wall is of Gram-negative architecture, as a central murein layer and an outer membrane were clearly discernible. No evidence for flagellation was observed, which is consistent with the observations from the original de- scription (Schauder \& Schink, 1989), namely, that the organism lost its motility after a period of cultivation.

\section{DNA base composition}

The DNA $\mathrm{G}+\mathrm{C}$ base composition of the three species of Anaerovibrio was reported to range from $31.5 \mathrm{~mol} \%$ for Anaerovibrio lipolytica (Schauder \& Schink, 1989) to $44 \cdot 1 \mathrm{~mol} \%$ for Anaerovibrio burkinabensis (Ouattara et al., 1992). Because values below $40 \mathrm{~mol} \%$ for $\mathrm{G}+\mathrm{C}$ content are not characteristic of species within the Sporomusa-Pectinatus-Selenomonas phyletic group, the wide range of values reported also calls into question the inclusion of these organisms into a single genus; this is especially true if the differences in $\mathrm{G}+\mathrm{C}$ content, the implied DNA cistron dissimilarity and the inferred potential evolutionary divergence (De Ley, 1967) are taken into account. While we were able to confirm the reported $\mathrm{G}+\mathrm{C}$ content of Anaerovibrio glycerini as $35.0 \mathrm{~mol} \%( \pm 0.3 \%, n=10)$, we found the value for Anaerovibrio burkinabensis to be $48.5 \mathrm{~mol} \%$ $( \pm 0.4 \%, n=5)$ instead of the reported $44.1 \mathrm{~mol} \%$. The $\mathrm{G}+\mathrm{C}$ content of Anaerovibrio lipolytica was determined to be $44.0 \mathrm{~mol} \%( \pm 0 \cdot 3 \%, n=4)$, which is significantly higher than the value of $31.5 \mathrm{~mol} \%$ reported previously.

\section{5 rDNA sequence analysis}

PCR amplification and subsequent direct cycle sequencing of the PCR product allowed the determination of the nucleotide sequence of the nearly complete $16 \mathrm{~S}$ rRNA gene of Anaerovibrio glycerini. However, a part of the sequence near the $5^{\prime}$-end of the 16S rRNA genes of Anaerovibrio lipolytica and Anaerovibrio burkinabensis, corresponding to helix 6 (Neefs et al., 1991), could not be resolved using this protocol. Apparently, sequence-inserts in helix 6 and multiple, heterogeneous $16 \mathrm{~S}$ rDNA operons are common throughout many members of the Sporomusa-Pectinatus-Selenomonas phyletic group. This is indicated by a relatively high degree of local sequence ambiguities in many published 16S rRNA sequences, or the omission of the $5^{\prime}$-end of published 16S rDNA sequences (Maidak et al., 1999; Schleifer et al., 1990; Stoesser et al., 1999), although secondary structure effects also may have influenced the sequence quality in some cases. The occurrence of long inserts in the 16S rRNA (Patel et al., 1992) and 16S rRNA genes (Rainey et al., 1996; Stackebrandt et al., 1997) has been demonstrated in various anaerobic organisms in which cloning and subsequent sequencing of the PCR-amplification products of the 16S rRNA genes led to the resolution of the full nucleotide sequences of the individual clones. In the case of the present study, the presence of multiple 16S rRNA genes of different length and/or sequence in Anaerovibrio lipolytica was confirmed by single-strand conformation polymorphism (SSCP) gel electrophoresis, as well as by cloning of the PCRamplification product and sequence determination of individual clones (unpublished results). 

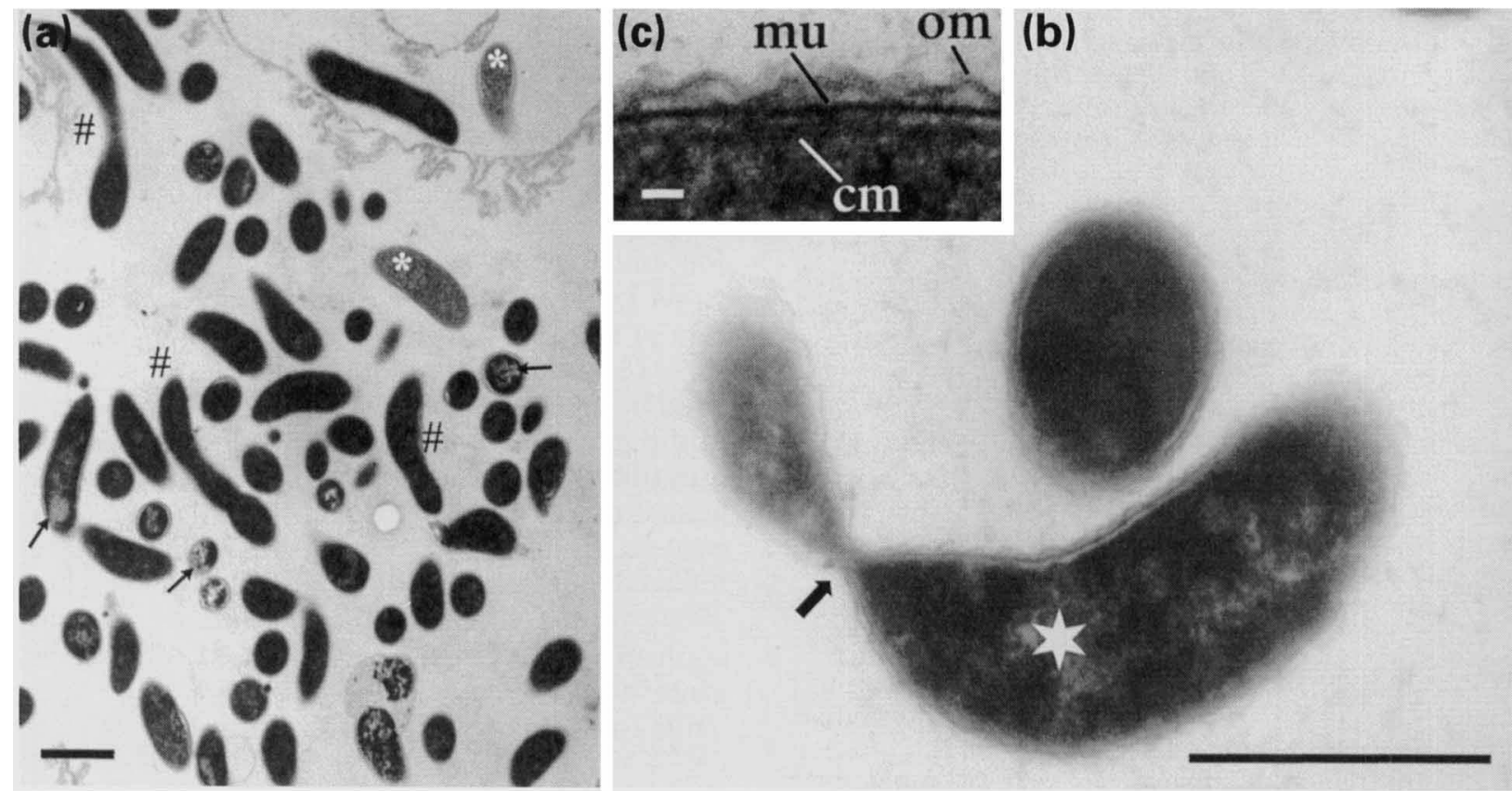

Fig. 1. Ultrastructure of Anaerovibrio glycerini. (a) General morphology. Cells show a characteristic vibrioform shape (\#). Generally, the cytoplasm appears to be rather electron-dense. Some cells, however, are electron-translucent (asterisks). The bacterial chromosome tends to form condensed centres (arrows), which can be identified because of their high electron-transparency. (b) Cell division. Upon cell division, the daughter cells taper off at the division plane (arrow). The star indicates electron-dense, loosely packed DNA in this cell. (c) Cell wall and membranes. The presence of an outer membrane (om) and a peptidoglycan layer $(\mathrm{mu})$ indicates that the cell wall is of the Gram-negative type $(\mathrm{cm}=$ cytoplasmic membrane). Bars, $1 \mu \mathrm{m}$ (a); $500 \mathrm{~nm}(\mathrm{~b}) ; 10 \mathrm{~nm}$ (c).

The lengths of the $16 \mathrm{~S}$ rDNA sequences of the strains analysed ranged from 1470 to 1550 nucleotides, i.e. equivalent to more than $97 \%$ of the estimated length of the respective genes. The sequences were aligned manually to reference sequences and, after the omission of non-homologous or ambiguously determined nucleotides, approximately 1290 nucleotide positions were used to calculate sequence similarities and evolutionary distances. Because of the incompleteness of available sequence data and the strain-specific sequence heterogeneity of operons within individual strains, the sequence region of helix 6 was omitted from the phylogenetic analysis. Comparisons with reference sequences from the RDP and GenBank/ EMBL/DDBJ databases revealed that the three species of the genus Anaerovibrio cluster within the radiation of the Sporomusa-Pectinatus-Selenomonas phyletic group, i.e. clostridial cluster IX, as described by Collins et al. (1994). Sequence comparisons revealed approximately $9-11 \%$ 16S rRNA gene sequence dissimilarity between the three species of the genus Anaerovibrio. A cluster analysis (Fig. 2) placed them in three different evolutionary lineages within the Sporomusa-Pectinatus-Selenomonas phyletic group. The similarity of the 16S rDNA sequence of Anaerovibrio lipolytica to those of Anaerovibrio glycerini and Anaerovibrio burkinabensis was observed to be 89.5 and $89.0 \%$, respectively. The sequence similarity between the 16S rRNA genes of Anaerovibrio glycerini and
Anaerovibrio burkinabensis was observed to be $90 \cdot 4 \%$. The rumen organism Anaerovibrio lipolytica clustered within a lineage including the species of the genera Selenomonas, Schwartzia, Centipeda and Mitsuokella (which primarily inhabit the mammalian gut and oral cavity) as well as species of the genera Pectinatus and Zymophilus (which cause beer-spoilage). The highest sequence-similarity values determined from comparisons of the 16S rDNA sequence of Anaerovibrio lipolytica with reference sequence data were $92-93 \%$, i.e. to the 16S rRNA gene sequences of Selenomonas ruminantium subsp. ruminantium, Mitsuokella multacida and Schwartzia succinivorans. Anaerovibrio burkinabensis was observed to be positioned on a distinct phylogenetic branch (Fig. 2), the closest relative being Zymophilus paucivorans, with a 16S rRNA sequence similarity of $91.0 \%$. Interestingly, Anaerovibrio glycerini was most closely related to spore-forming organisms such as the generically misnamed Clostridium quercicolum $(93.7 \%$ sequence similarity) and species of the genera Sporomusa and Acetonema.

\section{Respiratory lipoquinones}

All three members of the genus Anaerovibrio were shown to contain the compound previously reported as 'lipid F' (Schleifer et al., 1990; Stackebrandt et al., 1985). Structural studies on this compound indicate that it is a modified naphthoquinone with an iso- 


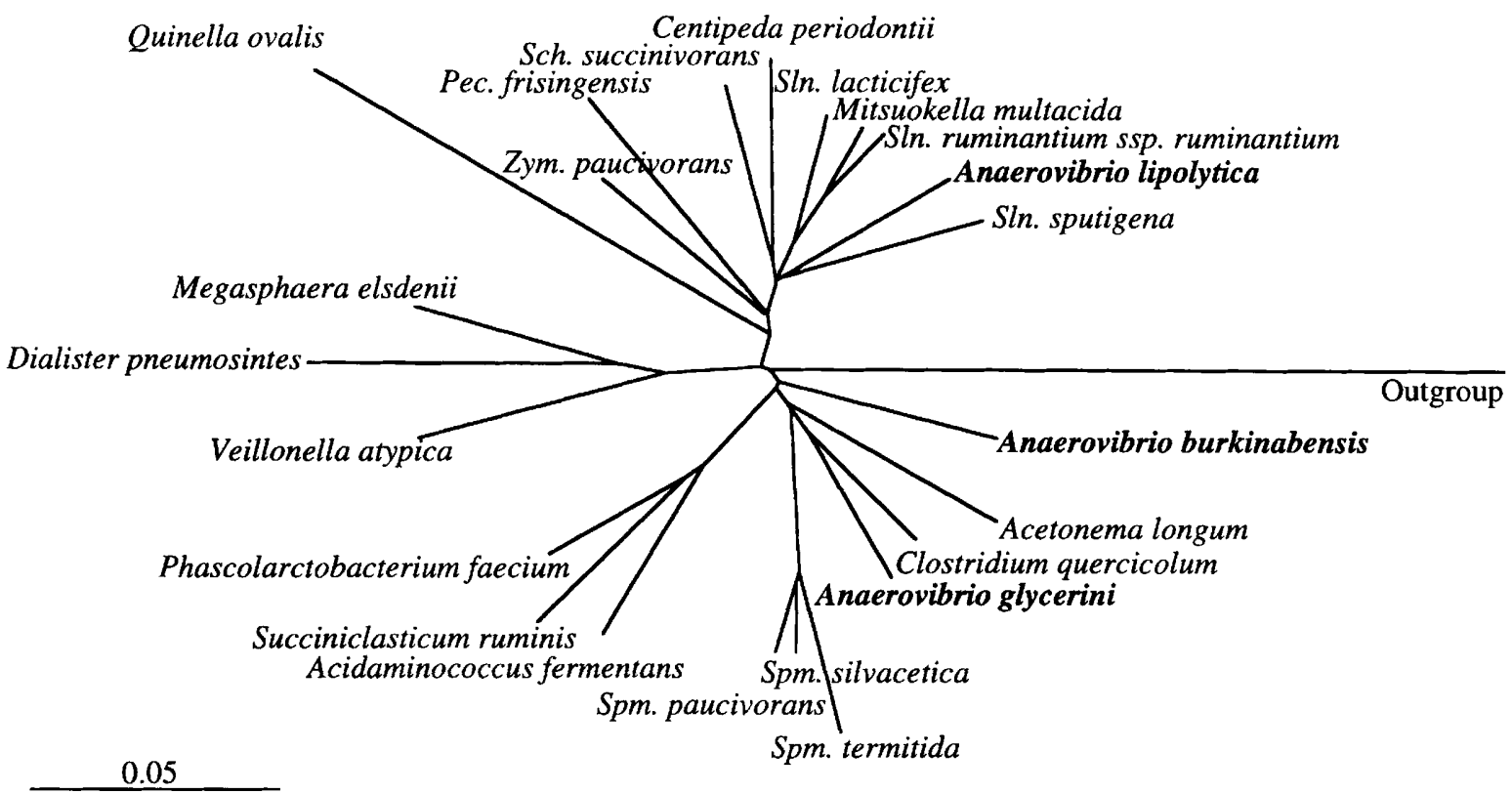

Fig. 2. Dendrogram showing the inferred phylogenetic relationships of the members of the genus Anaerovibrio with other genera in the Sporomusa sub-branch of the clostridia. Evolutionary distances were derived from pairwise sequence dissimilarities and the dendrogram was generated using the FITCH program of the PHYLIP package (Felsenstein, 1989). The sequences of the following strains were obtained from the GenBank/EMBLDDBJ and RDP databases or from the references given in parentheses: Selenomonas (Sin.) ruminantium subsp. ruminantium GA192 ${ }^{\top}\left(\mathrm{M}^{2} 2702^{\top}\right.$ ), Selenomonas lacticifex DSM $20757^{\top}$ (Schleifer et al., 1990), Selenomonas sputigena ATCC $38185^{\top}$ (Schleifer et al., 1990), Zymophilus (Zym.) paucivorans DSM $20756^{\top}$ (Schleifer et al., 1990), Pectinatus (Pec.) frisingensis ATCC 33332 ${ }^{\top}$ (Schleifer et al., 1990), Schwartzia (Sch.) succinivorans DSM $10502^{\top}$ (Y09434 ${ }^{\top}$ ), Acidaminococcus fermentans VP4 ${ }^{\top}$ (RDP), Phascolarctobacterium faecium ACM 3670 (X72865), Megasphaera elsdenii B159 ${ }^{\top}$ (RDP), Sporomusa (Spm.) paucivorans $\mathrm{X}^{\top}$ (RDP), Sporomusa silvacetica DG-1 ${ }^{\top}$ (Y09976), Sporomusa termitida JSN-2 ${ }^{\top}$ (M61920), Succiniclasticum ruminis SE10 ${ }^{\top}$ (X81137), Veillonella atypica DSM 20739 ${ }^{\top}$ (X84007), Quinella ovalis (RDP), Dialister pneumosintes ATCC $33048^{\top}$ (X82500), Mitsuokella multacida NCTC $10934^{\top}$ (X81878). Bar, 5 inferred nucleotide substitutions per 100 positions.

Table 1. Relative proportions (\%) of 'lipid F' isoprenologues in Anaerovibrio lipolytica (DSM 3074'), Anaerovibrio glycerini (DSM 5192') and Anaerovibrio burkinabensis (DSM 6283')

\begin{tabular}{|lcccc|}
\hline Species & \multicolumn{4}{c|}{$\begin{array}{c}\text { No. isoprenoid units attached to } \\
\text { quinone nucleus: }\end{array}$} \\
\cline { 2 - 5 } & $\mathbf{7}$ & $\mathbf{8}$ & $\mathbf{9}$ & $\mathbf{1 0}$ \\
\hline A. lipolytica & 2.9 & 92.4 & $4 \cdot 7$ & - \\
A. glycerini & $2 \cdot 5$ & $29 \cdot 8$ & $60 \cdot 4$ & $7 \cdot 3$ \\
A. burkinabensis & $5 \cdot 5$ & 73.9 & $19 \cdot 5$ & $1 \cdot 1$ \\
\hline
\end{tabular}

prenoid side chain (B. J. Tindall, V. Wray \& H. Hippe, unpublished results). The relative percentage composition of the 'lipid $F$ ' isoprenologues is given in Table 1. It is interesting to note that, in Anaerovibrio lipolytica, the major component is 'lipid F' with eight isoprenologues, accompanied by smaller amounts of the hepta- and nonaprenologue. In Anaerovibrio burkinabensis, the octa- and nonaprenologues predominate in the ratio $4: 1$, with smaller amounts of hepta- and decaprenologue being present. In Anaerovibrio gly- cerini, the major components are the octa- and nonaprenalogues, in the ratio $1: 2$. No evidence was found for the presence of ubiquinones or menaquinones.

\section{Polar lipids}

All three species of Anaerovibrio examined had a characteristic polar lipid pattern (Fig. 3) in which only two phospholipids predominated, namely phosphatidyl serine and phosphatidyl ethanolamine. This observation is consistent with reports that these two compounds are the dominant phospholipids in $A n$ aerovibrio lipolytica, Veillonella parvula, Megasphaera elsdenii and Selenomonas ruminantium (Johnston \& Goldfine, 1982; Kamio \& Takahashi, 1980; Prins et al., 1974; van Golde et al., 1973; Verkley et al., 1975; Watanabe et al., 1982). Shah et al. (1983) also reported a similar lipid composition for Megamonas hypermegale and Mitsuokella multacida, although these authors did not unambiguously identify the second amino-phospholipid as phosphatidyl serine. Although phosphatidyl serine is generally assumed to be a precursor of phosphatidyl ethanolamine (which is formed by decarboxylation of phosphatidyl serine) in bacteria, the former compound is rarely found in such large quantities as those observed in members of the 


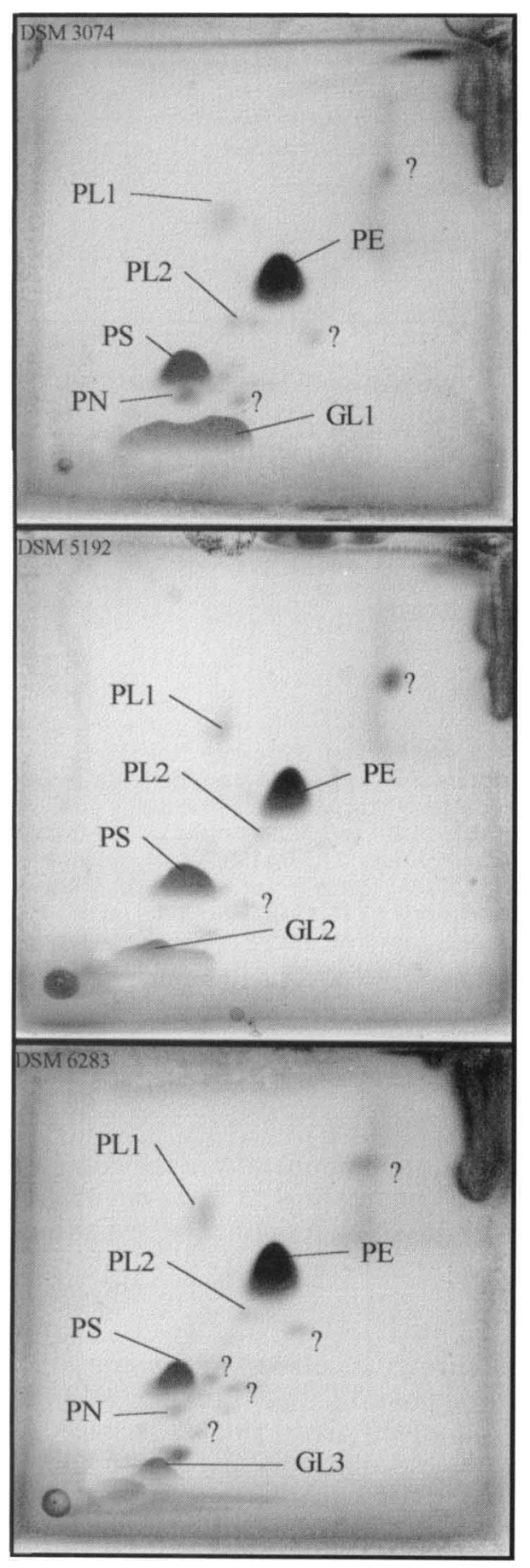

Fig. 3. Two-dimensional thin-layer chromatograms of the polar lipids of Anaerovibrio lipolytica (DSM 3074'), Anaerovibrio glycerini (DSM 5192 ${ }^{\top}$ ) and Anaerovibrio burkinabensis (DSM $\left.6283^{\mathrm{T}}\right)$; for details, see Methods. PE, phosphatidyl ethanolamine; PS, phosphatidyl serine; PN, amino-phospholipid; PL1 and PL2, unidentified phospholipids; GL1, GL2 and GL3, unidentified glycolipids showing atypical staining behaviour; ?, material staining in a characteristic fashion with the anisaldehyde/sulfuric acid reagent, but which could not be assigned a chemical structure.
Sporomusa-Pectinatus-Selenomonas phyletic group. Recent reports of the lipid composition of the fusobacteria (Sadek et al., 1998) show that these organisms synthesize phosphatidyl ethanolamine and phosphatidyl glycerol. A survey of a wide range of bacteria indicates that the presence of only phosphatidyl serine and phosphatidyl ethanolamine as the dominant polar lipids is a feature that further points to the unique evolutionary and taxonomic status of the Sporomusa-Pectinatus-Selenomonas phyletic group within the Bacteria. The only organisms in which phosphatidyl serine is reported to be synthesized in significant quantities, albeit in the form of di- and tetraether derivatives, are ones found in the Archaea, within the phyletic groups defined by the genera Methanosarcina/Methanolobus, Methanobacterium/ Methanobrevibacter/Methanosphaera and Methanococcus (Koga et al., 1993).

A third lipid was also visible in the polar lipids of all Anaerovibrio species examined, as also reported by Shah et al. (1983). The staining behaviour of this compound indicated that it may be an atypical glycolipid. An unsual glycolipid was also reported in Selenomonas ruminantium by Kamio et al. (1972a). The exact identification of this compound in the Anaerovibrio species requires further detailed structural work.

\section{Fatty acids}

The application of a differential hydrolysis method to the detection of fatty acids and other long-chain hydrocarbons (i.e. dimethyl acetals, Fig. 4a) provided interesting results for the three Anaerovibrio species examined. Several components were shown to increase their relative percentage composition when subjected to the differential hydrolysis methodology. Such behaviour is not typical of ester-linked fatty acids, nor of plasmalogens (which give rise to dimethyl acetals). Despite the fact that Moore et al. (1994) previously reported the presence of fairly large amounts of dimethyl acetals in Anaerovibrio lipolytica and other members of the Sporomusa-Pectinatus-Selenomonas phyletic group, our preliminary results cast doubt on the presence of large amounts of dimethyl acetals. Examination of the fatty acid composition of all three species of the genus Anaerovibrio showed that fatty acids predominated. In addition, 3-hydroxy fatty acids could be identified in all strains by GC-MS, on the basis of the characteristic fragmentation of the side chain at $m / z 103$ (Fig. 4b). This is in contrast to the work of Moore et al. (1994), where dimethyl acetals and relatively small amounts of an unresolved mixture of 15:0 dimethyl acetal/3-OH 14:0 fatty acid were reported for Anaerovibrio lipolytica. In order to confirm the presence of 3-hydroxy fatty acids in the three species of Anaerovibrio the fatty acids were subjected to trimethylsilation, which results in the formation of trimethylsilyl derivatives of hydroxy fatty acids. The following facts were taken as conclusive evidence that the compounds were 3-hydroxy fatty 
(a)

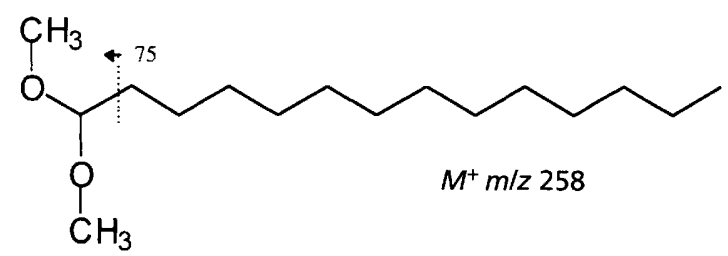

(b)<smiles>CCCCCCCCCCC(O)[CH+]C(=O)OC</smiles>

(c)

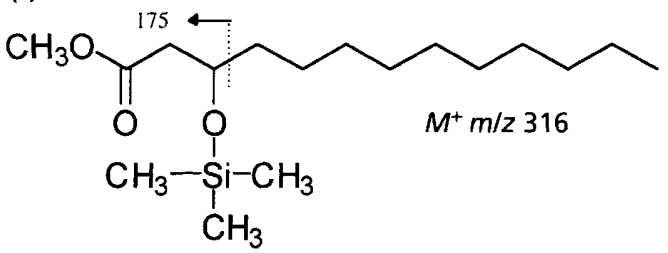

Fig. 4. Structures of (a) 14:0 dimethyl acetal, (b) $3-\mathrm{OH} 13: 0$ fatty acid and (c) the trimethylsilyl derivate of 3-OH 13:0 fatty acid, indicating their $\mathrm{M}^{+}$ions and the major fragments due to the presence of the dimethyl acetal or 3-hydroxyl groups, respectively (dotted lines to which the molecular mass $\mathrm{m} / \mathrm{z}$ has been assigned).

acids: (1) the presumptive 3-hydroxy fatty acids showed a shift in retention time because of the formation of trimethylsilyl derivatives; (2) the molecular mass increased by 72 mass units (owing to the formation of trimethylsilyl derivatives); (3) a characteristic fragment formed at $m / z \quad 175$ (because of fragmentation of the trimethylsilyl derivative of a 3hydroxy fatty acid) (Fig. 4c). Furthermore, differential hydrolysis indicated that about half of the 3-hydroxy fatty acids were amide linked, a finding consistent with the reports of a similar distribution of amide-linked 3hydroxy fatty acids in members of the genus Pectinatus and Selenomonas (Helander et al., 1994; Kamio et al., 1972a, b). Examination of the MIDI database, which was used to evaluate the data of Moore et al. (1994), indicates that the 3-OH 13:0 fatty acid is not present in the anaerobe database. In the aerobe database, the 3OH 13:0 fatty acid is present, but dimethyl acetals are not listed. Comparison of the equivalent chain-length data in the two databases indicates that the 3-OH 13:0 fatty acid and 14:0 dimethyl acetal cannot be resolved under the chromatographic conditions used and so should be recorded as an unresolved peak. However, identification based on the present databases identifies the same compound as a 14:0 dimethyl acetal in the anaerobe database but as a 3-OH 13:0 fatty acid in the aerobe database. We suggest, based on our data that Moore et al. (1994) wrongly identified a 3-OH 13:0 fatty acid as a 14:0 dimethyl acetal. This would also explain why Miyagawa et al. (1979) and Shah et al. (1983) found 16-20\% 3-OH 13:0 fatty acid in Megamonas hypermegale, whereas Moore et al. (1994) reported $20 \% 14: 0$ dimethyl acetal in the same organism. However, our results contrast strongly with the finding of plasmalogens in a variety of species within the members of the Sporomusa-PectinatusSelenomonas phyletic group (Johnston \& Goldfine, 1982; Kamio \& Takahashi, 1980; Prins et al., 1974; van Golde et al., 1973; Verkley et al., 1975; Watanabe et al., 1982), including Anaerovibrio lipolytica. The chain lengths of the degradation products of the plasmalogens differ from those reported by Moore et al. (1994); further work is needed to clarify this point.

In all three species of the genus Anaerovibrio examined, the major fatty acids present were straight-chain, evenand odd-numbered, saturated and unsaturated fatty acids (Table 2). However, Anaerovibrio burkinabensis was clearly different from the other two species in that it also contained iso-branched fatty acids. All strains contained 3-OH fatty acids, a significant proportion of which was only released by stronger hydrolytic methods, suggesting that both ester- and amide-bound hydroxy fatty acids were present, consistent with the reports of amide-bound fatty acids in Pectinatus and Selenomonas species (Helander et al., 1994; Kamio et al., 1972a, b). In Anaerovibrio lipolytica the major 3$\mathrm{OH}$ fatty acids were 3-OH 13:0 and 3-OH 15:0, while in Anaerovibrio glycerini 3-OH 11:0, 3-OH 12:0 and 3$\mathrm{OH} 13: 0$ predominated. The presence of 3-OH 11:0, 3-OH 12:0, 3-OH iso-13:0 and 3-OH 13:0 in Anaerovibrio burkinabensis allowed all three species to be distinguished from one another on the basis of the 3hydroxy fatty acid composition. Close examination of the qualitative and quantitative distribution of the fatty acids $13: 0,15: 0,16: 0$ and $17: 0$, together with the various isomers of $13: 1,15: 1,16: 1$ and $17: 1$, provided additional data in support of the chemical distinctiveness of the three species.

\section{DISCUSSION}

Comparison of the 16S rDNA sequences of the type strains of the three described species constituting the genus Anaerovibrio revealed that they belong to the Sporomusa-Pectinatus-Selenomonas phyletic group. However, data from this study show a low degree of similarity of $16 \mathrm{~S}$ rDNA sequences, as well as significant differences in 'lipid F' composition (Table 1) and fatty acid profiles (Table 2) among the three organisms. Furthermore, a review of the available literature revealed that significant differences exist with respect to the metabolic traits of the three strains. The type species of the genus, Anaerovibrio lipolytica, clusters phylogenetically with the species of other genera from the rumen and other mesophilic ecosystems. This organism can be distinguished from members of the beer-spoiling genera Zymophilus and Pectinatus by their inability to grow on glycerol and their lower 
Table 2. Fatty acid composition of Anaerovibrio lipolytica (DSM 3074'), Anaerovibrio glycerini (DSM 5192') and Anaerovibrio burkinabensis (DSM 6283')

Values are given as percentage area.

\begin{tabular}{|c|c|c|c|c|c|c|}
\hline \multirow[t]{2}{*}{ Fatty acid } & \multicolumn{2}{|c|}{ A. lipolytica } & \multicolumn{2}{|c|}{ A. glycerini } & \multicolumn{2}{|c|}{ A. burkinabensis } \\
\hline & Method 1 & Method 2 & Method 1 & Method 2 & Method 1 & Method 2 \\
\hline $9: 0$ & & & $2 \cdot 2$ & & & \\
\hline $10: 0$ & & & $3 \cdot 2$ & $3 \cdot 4$ & & \\
\hline$?$ & & $0 \cdot 3$ & & & & \\
\hline i11:0 & & & & & $13 \cdot 0$ & $10 \cdot 4$ \\
\hline $11: 0$ & $7 \cdot 4$ & $5 \cdot 5$ & $4 \cdot 2$ & $3 \cdot 3$ & & \\
\hline $12: 0$ & 0.9 & 0.7 & & & & \\
\hline 3-OH-11:0 & & & $4 \cdot 6$ & $4 \cdot 8$ & $1 \cdot 3$ & 1.0 \\
\hline$?$ & & & $1 \cdot 4$ & $1 \cdot 0$ & & \\
\hline$?$ & & & & & & $0 \cdot 6$ \\
\hline $13: 1$ & $1 \cdot 0$ & $0 \cdot 7$ & & & & \\
\hline$?$ & & $0 \cdot 3$ & & & & \\
\hline $\begin{array}{l}13: 0 \\
?\end{array}$ & $4 \cdot 9$ & $3 \cdot 8$ & & & $2 \cdot 0$ & 3.7 \\
\hline $3-\mathrm{OH}-12: 0$ & & & $2 \cdot 2$ & $4 \cdot 7$ & $2 \cdot 0$ & $1 \cdot 1$ \\
\hline$?$ & $1 \cdot 1$ & $3 \cdot 0$ & & $1 \cdot 4$ & & $1 \cdot 1$ \\
\hline $14: 0$ & & & $1 \cdot 4$ & $1 \cdot 2$ & & \\
\hline $3-\mathrm{OH}-\mathrm{i} 13: 0$ & & & & & $5 \cdot 0$ & $11 \cdot 8$ \\
\hline$?$ & & 0.8 & & & & \\
\hline$?$ & & & & & $1 \cdot 3$ & $1 \cdot 1$ \\
\hline 3-OH-13:0 & $8 \cdot 8$ & $14 \cdot 5$ & $1 \cdot 4$ & $8 \cdot 1$ & & $2 \cdot 1$ \\
\hline$?$ & & 0.6 & & & & \\
\hline$?$ & & & & & & 0.8 \\
\hline $\begin{array}{l}15: 1 \\
15 \cdot 1\end{array}$ & & $\begin{array}{l}0.6 \\
7.6\end{array}$ & $17 \cdot 1$ & $14 \cdot 6$ & $16 \cdot 7$ & $13 \cdot 4$ \\
\hline $\begin{array}{l}15: 1 \\
15: 1\end{array}$ & $9 \cdot 7$ & $7 \cdot 6$ & $1 \cdot 0$ & $0 \cdot 8$ & 1.6 & 1.4 \\
\hline $\begin{array}{l}15: 1 \\
15: 0\end{array}$ & 18.0 & $14 \cdot 3$ & $17 \cdot 1$ & $15 \cdot 2$ & $5 \cdot 7$ & $5 \cdot 1$ \\
\hline ? & & & & & & $0 \cdot 7$ \\
\hline $3-\mathrm{OH}-14: 0$ & & $0 \cdot 7$ & & & & \\
\hline$?$ & & 0.9 & & & & \\
\hline $16: 1$ & $6 \cdot 8$ & $5 \cdot 3$ & & & $2 \cdot 9$ & $2 \cdot 8$ \\
\hline $16: 1$ & 1.9 & $1 \cdot 7$ & $4 \cdot 0$ & 3.5 & $4 \cdot 4$ & $3 \cdot 6$ \\
\hline $16: 1$ & & $0 \cdot 7$ & $4 \cdot 8$ & $4 \cdot 3$ & $4 \cdot 5$ & $3 \cdot 6$ \\
\hline$?$ & 1.5 & 1.5 & & & & \\
\hline $16: 0$ & 1.9 & $1 \cdot 7$ & $4 \cdot 3$ & $4 \cdot 0$ & $2 \cdot 1$ & $2 \cdot 2$ \\
\hline$?$ & & 0.9 & & & & \\
\hline i17: 1 & & & & & $7 \cdot 9$ & $6 \cdot 9$ \\
\hline 3-OH-15:0 & 1.5 & $2 \cdot 8$ & & & & \\
\hline$?$ & & & & & $1 \cdot 0$ & $1 \cdot 0$ \\
\hline $17: 1$ & $6 \cdot 7$ & $5 \cdot 4$ & $4 \cdot 8$ & $4 \cdot 7$ & $5 \cdot 0$ & $4 \cdot 6$ \\
\hline $17: 1$ & $15 \cdot 6$ & $13 \cdot 1$ & $15 \cdot 5$ & $14 \cdot 3$ & $18 \cdot 0$ & $16 \cdot 2$ \\
\hline $17: 1$ & & $0 \cdot 5$ & $1 \cdot 2$ & $1 \cdot 1$ & $2 \cdot 0$ & $1 \cdot 8$ \\
\hline $17: 0$ & $8 \cdot 5$ & $7 \cdot 6$ & 8.6 & $8 \cdot 7$ & $2 \cdot 4$ & $2 \cdot 5$ \\
\hline $18: 1$ & $1 \cdot 0$ & $0 \cdot 8$ & $1 \cdot 1$ & $1 \cdot 1$ & $1 \cdot 1$ & $0 \cdot 8$ \\
\hline$?$ & & 0.5 & & & & \\
\hline $19: 1$ & $1 \cdot 3$ & $1 \cdot 2$ & & & & \\
\hline 19:1 & 1.8 & $1 \cdot 6$ & & & & \\
\hline 19:0 & & 0.7 & & & & \\
\hline
\end{tabular}

?, Unknown structure.

Method 1 and Method 2 refer to the differential release of fatty acids, which indicates the presence of ester- and (presumptive) amide- plus ester-linked fatty acids. 
temperature and $\mathrm{pH}$ optima for growth. Instead, species of these genera utilize a wide range of sugars that cannot be used by Anaerovibrio lipolytica. Additionally, members of the genus Pectinatus possess a unique type of flagellation, described as comb-like, while Anaerovibrio lipolytica, like many Selenomonas species, possesses a tuft of flagella on the concave side of the cell (Henderson \& Hodgkiss, 1973). Species of the genus Selenomonas can be distinguished from species of the genus Anaerovibrio on the basis of their different fermentation profiles and a generally higher genomic DNA G + C content. The relatively fastidious species Selenomonas acidaminophila, although having a genomic DNA $\mathrm{G}+\mathrm{C}$ content similar to that of Anaerovibrio lipolytica, does not grow on glycerol and utilizes glutamate, aspartate and pyruvate, none of which is fermented by Anaerovibrio lipolytica. The recently described species Selenomonas lipolytica (Dighe et al., 1998), although having in common with Anaerovibrio lipolytica the ability to carry out lipolysis and a similar $\mathrm{G}+\mathrm{C}$ content of the genomic DNA, differs in its natural habitat, its much wider substrate spectrum and its $16 \mathrm{~S}$ rDNA sequence. The published partial $16 \mathrm{~S}$ rDNA sequence of $S$. lipolytica is $90.9 \%$ similar to the sequence of the type strain of $S$. ruminantium, but is only $82.4 \%$ similar to the sequence of Anaerovibrio lipolytica. Mitsuokella multacida, the non-motile, rod-shaped organism isolated from human and pig faeces, differs from Anaerovibrio lipolytica in the wide range of sugars used as substrates, the inability to ferment glycerol and the different fermentation products. It also has a much higher $\mathrm{G}+\mathrm{C}$ content in the genomic DNA. Another organism showing a high degree of $16 \mathrm{~S}$ rDNA sequence similarity to Anaerovibrio lipolytica, the recently described rumen organism Schwartzia succinivorans (van Gylswyk et al., 1997), is a substrate specialist that uses succinate as the sole energy source. This bacterium was reported to have a genomic DNA G $+\mathrm{C}$ content of $46 \mathrm{~mol} \%$, which is similar to that of Anaerovibrio lipolytica $(44 \mathrm{~mol} \%)$. However, from the available genetic and biochemical data, and because of the different ecological niche it occupies in the rumen ecosystem, Schwartzia succinivorans belongs to a different genus from that of Anaerovibrio lipolytica. The $16 \mathrm{~S}$ rDNA sequence data suggest that Anaerovibrio lipolytica shares a common ancestor with species of the genus Selenomonas and related genera.

Anaerovibrio glycerini is highly specialized in the fermentation of glycerol and the glycerol moeity in diolein. This organism is not able to hydrolyse neutral fats, this being a key feature of the genus Anaerovibrio (Prins, 1986). It is also unable to grow on sugars or organic acids, has a lower temperature optimum and has a lower $\mathrm{G}+\mathrm{C}$ content in the genomic DNA than the type species, Anaerovibrio lipolytica. Anaerovibrio glycerini differs from species of the genus Sporomusa by the inability to gain energy from most substrates that have been described as being utilized by species of the genus Sporomusa, and by the inability to grow chemoautotrophically. In addition, Anaerovibrio glycerini, unlike species of the genus Sporomusa, does not form spores, is catalase-negative and produces molecular hydrogen. Furthermore, Anaerovibrio glycerini differs from the non-sporulating species Sporomusa paucivorans in terms of the genomic DNA $\mathrm{G}+\mathrm{C}$ content, the substrate spectrum and the lack of motility. The organism showing the greatest degree of 16S rDNA sequence similarity, Clostridium quercicolum, is a morphologically distinct spore-former with a much higher genomic DNA $\mathrm{G}+\mathrm{C}$ content (52-54 mol\%, Stankewich et al., 1971) than Anaerovibrio glycerini $(35 \mathrm{~mol} \%$ ).

Anaerovibrio burkinabensis differs from the type species of the genus Anaerovibrio, not only in its 16S rDNA sequence, lipid patterns and 'lipid $F$ ' profile, but also in the type of flagellation and in the wider range of substrates utilized. Anaerovibrio burkinabensis does not ferment glycerol and is non-lipolytic. It can be differentiated phenotypically from other vibrioid genera in the Sporomusa-Pectinatus-Selenomonas phyletic group by the type of flagellation, the inability to form spores and also by its unique substrate spectrum.

The presence of 'lipid F' in the three species of the genus Anaerovibrio examined is consistent with that which has already been published on the presence of this unique compound in members of the SporomusaPectinatus-Selenomonas phyletic group (Möller et al., 1984; Schleifer et al., 1990; Stackebrandt et al., 1985). Although Möller et al. (1984) initially reported that ubiquinones were present in members of the genus Sporomusa, this was subsequently corrected (Stackebrandt et al., 1985). Shah et al. (1983) reported that Mitsuokella multacida (basonym: Bacteroides multiacidus) and Megamonas hypermegale (basonym: Bacteroides hypermegas), both of which share chemical characteristics with members of the Sporomusa-Pectinatus-Selenomonas phyletic group, did not contain ubiquinones or menaquinones, although the presence of 'lipid F' cannot be excluded. Detailed published data on the distribution of different isoprenologues of 'lipid F' in members of this phyletic group are lacking, but Kroppenstedt et al. (1985) presented an overview of the taxonomic significance of different isoprenologues. In general, it can be concluded that the presence of 'lipid $F$ ' is a feature common to all members of this phyletic group that have been examined for its presence, and that the distribution of the different isoprenologues is a useful taxonomic marker. It is possible to unambiguously distinguish each of the three species within the genus Anaerovibrio, not only from the qualitative distribution of the isoprenologues of 'lipid F', but also on the basis of their relative distribution. Such variations in isoprenoid quinone distribution are generally taken to indicate differences in taxonomic rank at or above genus level. We conclude, therefore, that the presence of qualitative and quantitative differences in the 
distribution of the different isoprenologues of 'lipid F' is indicative of the fact that the three species of the genus Anaerovibrio cannot be maintained in the same genus.

Examination of the fatty acid composition of the three Anaerovibrio species showed that they could be distinguished from one another on the basis of fatty acid composition alone. It is also interesting to note that, after correcting the misidentification of $3-\mathrm{OH}$ fatty acids in the work of Moore et al. (1994) (for a discussion, see the Results above), it is possible to detect a general fatty acid pattern common to all members of the Sporomusa-Pectinatus-Selenomonas phyletic group examined to date (Miyagawa et al., 1979; Moore et al., 1994; Shah et al., 1983). The fatty acids are straight, saturated or unsaturated chains; in some species iso-branched fatty acids are present in significant quantitities; $10-20 \%$ of the fatty acids are either odd-numbered (C-11 or C-13) or evennumbered (C-12), while $\mathrm{C}-15 / \mathrm{C}-17$ or $\mathrm{C}-16 / \mathrm{C}-18$ account for $30-50 \%$ of the fatty acids. All organisms contain relatively large amounts of 3-hydroxy fatty acids, in which the dominant compound has 13 or 14 carbon atoms (i.e. $3-\mathrm{OH} 13: 0 / 3-\mathrm{OH}$ iso-13:0 or $3-\mathrm{OH}$ 14:0). It is interesting to note that, despite their apparent 16S rDNA sequence association with the clostridia and their relatives, members of the Sporomusa-Pectinatus-Selenomonas phyletic group appear to have an evolutionary relationship with certain members of the fusobacteria (Jantzen \& Hofstad, 1981): they share the unusual feature of being Gramnegative and having significant quantities of hydroxylated fatty acids in their outer cell layers, which appear to be associated with lipopolysaccharides.

In summary, the chemical composition of those species within members of the Sporomusa-PectinatusSelenomonas phyletic group examined to date indicates a common pattern, to which the members of the genus Anaerovibrio also conform. The chemical data presented provide independent evidence delineating this phyletic group and it is clear that the three species within the genus Anaerovibrio, as currently defined, are sufficiently different to be placed in different genera. We believe that the combination and integration of phenotypic and genotypic data provides a useful vehicle for examining the evolutionary and taxonomic relationships and diversity of prokaryotes. Within the framework of the present study, we have provided evidence for the taxonomic rearrangement of the members of the genus Anaerovibrio based on this principle. We propose that the two species Anaerovibrio glycerini and Anaerovibrio burkinabensis should be assigned to two new genera, as Anaerosinus glycerini gen. nov., comb. nov. and Anaeroarcus burkinensis [corrig.] gen. nov., comb. nov., respectively. According to Rule 65 of the Bacteriological Code (1990 Revision) (Lapage et al., 1992), the name Anaerovibrio lipolytica Hungate 1966 is corrected to Anaerovibrio lipolyticus [corrig.].

\section{Description of Anaerosinus gen. nov.}

Anaerosinus [An.ae.ro.si'nus. Gr. pref. an not; Gr. n. aer air; anaero not (living) in air; L. masc. n. sinus bend; M.L. masc. n. Anaerosinus a curved organism not living in air].

Cells are curved rods or spirals exhibiting a Gramnegative cell wall. Motile, but motility may be lost in culture. Endospores are not formed. Obligately anaerobic. Chemo-organotrophic. Catalase-negative. Cytochrome $b$ and 'lipid F', with octa- and nonaprenologues (ratio $1: 2$ ) as the predominating isoprenologues, are present. Mesophilic. The $\mathrm{G}+\mathrm{C}$ content of the DNA is approximately $35 \mathrm{~mol} \%$. The type species is Anaerosinus glycerini.

\section{Description of Anaerosinus glycerini (Schauder and Schink 1989) comb. nov.}

The description of Anaerosinus glycerini is identical to that given for Anaerovibrio glycerini (Schauder \& Schink, 1989). The type strain is DSM $5192^{\mathrm{T}}$.

\section{Description of Anaeroarcus gen. nov.}

Anaeroarcus [An.ae.ro.ar'cus. Gr. pref. an not; Gr. n. aer air; anaero not (living) in air; L. masc. n. arcus a bow, arc; Anaeroarcus a bow not living in air].

Cells are curved or spiral-shaped rods, motile and Gram-negative. Endospores are not formed. Obligately anaerobic chemo-organotroph. A limited range of organic acids, amino acids, carbohydrates and alcohols are fermented mainly to acetate, propionate, succinate and propanol. Yeast extract, Casamino acids, peptone and Biotrypticase support growth. 'Lipid F', with octa- and nonaprenologues (ratio 4:1) as the predominating isoprenologues, and cytochrome $b$ are present. Ferric iron, but not sulfate or nitrate, is reduced,. Mesophilic. The $\mathrm{G}+\mathrm{C}$ content of the genomic DNA is approximately $48 \mathrm{~mol} \%$. The type species is Anaeroarcus burkinensis [corrig.].

\section{Description of Anaeroarcus burkinensis (Ouattara, Traore and Garcia 1992) [corrig.] comb. nov.}

Anaeroarcus burkinensis (bur.kin.en'sis. N.L. adj. burkinensis pertaining to Burkina Faso, the place from which the organism was isolated; the specific epithet burkinabensis, as originally derived, has been corrected because the Latin suffix -ensis is used only to indicate geographic locations, not the inhabitants).

The description of Anaeroarcus burkinensis is identical to that given for Anaerovibrio burkinabensis (Ouattara et al., 1992). The type strain is DSM $6283^{\mathrm{T}}$.

\section{ACKNOWLEDGEMENTS}

We gratefully acknowledge the excellent technical assistance of Annette Krüger (16S rRNA gene sequencing) and Elke Haase (sample preparation for electron microscopy). Chris- 
tian Hesse and Jutta Burghardt are acknowledged for supporting the $\mathrm{G}+\mathrm{C}$ analyses. Thanks is given to an anonymous reviewer, who advised us to correct the specific epithet burkinabensis. This study was supported, in part, by funding from the German Federal Ministry for Science, Education and Research (project no. 0319433C) and through the German/New Zealand Science and Technology Cooperation Project (NEU-015-96). G. N.J. was the recipient of a travel fellowship from the DAAD (German Academic Exchange Service).

\section{REFERENCES}

Collins, M. D., Lawson, P. A., Willems, A., Cordoba, J. J., Fernandez-Garayzabal, J., Garcia, P., Cai, J., Hippe, H. \& Farrow, J. A. E. (1994). The phylogeny of the genus Clostridium: proposal of five new genera and eleven new species combinations. Int $J$ Syst Bacteriol 44, 812-826.

De Ley, J. (1967). Molecular biology and bacterial phylogeny. In Evolutionary Biology, pp. 103-156. Edited by T. Dobzhansky, M. K. Hecht \& W. C. Steere. New York: Plenum Press.

Dighe, A. S., Shouche, Y. S. \& Ranade, D. R. (1998). Selenomonas lipolytica sp. nov., an obligately anaerobic bacterium possessing lipolytic activity. Int $J$ Syst Bacteriol 48, 783-791.

Felsenstein, J. (1989). PHYLIP - Phylogeny inference package (version 3.2). Cladistics 5, 164-166.

van Golde, L. M. G., Prins, R. A., Franklin-Klein, W. \& AkkermansKryswijk, J. (1973). Phosphatidylserine and its plasmalogen analogue as major lipid constituents in Megasphaera elsdenii. Biochim Biophys Acta 326, 314-323.

Gutell, R. R., Weiser, B., Woese, C. R. \& Noller, H. F. (1985). Comparative anatomy of $16 \mathrm{~S}$-like ribosomal RNA. Prog Nucleic Acid Res Mol Biol 32, 155-216.

van Gylswyk, N. O., Hippe, H. \& Rainey, F. A. (1997). Schwartzia succinivorans gen. nov., sp. nov., another ruminal bacterium utilizing succinate as the sole energy source. Int J Syst Bacteriol 47, 155-159.

Helander, I. M., Kilpeläinen, I.-V., Moran, A. P., Lindner, B. \& Seydel, U. (1994). Chemical structure of the lipid A component of lipopolysaccharides of the genus Pectinatus. Eur J Biochem 224, 63-70.

Henderson, C. \& Hodgkiss, w. (1973). An electron microscopic study of Anaerovibrio lipolytica (Strain 5S) and its lipolytic enzyme. J Gen Microbiol 76, 389-393.

Hobson, P. N. \& Mann, S. O. (1961). The isolation of glycerolfermenting and lipolytic bacteria from the rumen of the sheep. $J$ Gen Microbiol 25, 227-240.

Holdeman, L. V., Cato, E. P. \& Moore, W. E. C. (1977). Anaerobe Laboratory Manual, 4th edn. Blacksburg, VA: Virginia Polytechnic Institute and State University.

Hungate, R. E. (1966). The Rumen and its Microbes. New York: Academic Press.

Jantzen, E. \& Hofstad, T. (1981). Fatty acids of Fusobacterium species: taxonomic implications. J Gen Microbiol 123, 163-171.

Johnston, N. C. \& Goldfine, H. (1982). Effects of growth temperature on fatty acid and alk-1-enyl group compositions of Veillonella parvula and Megasphaera elsdenii phospholipids. $J$ Bacteriol 149, 567-575.

Jukes, T. H. \& Cantor, C. R. (1969). Evolution of protein molecules. In Mammalian Protein Metabolism, pp. 21-132. Edited by H. H. Munro. New York: Academic Press.

Kamio, Y. \& Takahashi, H. (1980). Isolation and characterisation of outer and inner membranes of Selenomonas ruminantium. $J$ Bacteriol 141, 888-898.

Kamio, Y., Kim, K. C. \& Takahashi, H. (1972a). Identification of the basic structure of a glycolipid from Selenomonas ruminantium: as $\beta$-glucosaminyl-1,6-glucosamine. Agric Biol Chem 12 , 2195-2201.

Kamio, Y., Kim, K. C. \& Takahashi, H. (1972b). Characterisation of lipopolysaccharides of Selenomonas ruminantium. Agric Biol Chem 13, 2425-2432.

Karlson, U., Dwyer, D. F., Hooper, S. W., Moore, E. R. B., Timmis, K. N. \& Eltis, L. D. (1993). Two independently regulated cytochromes P-450 in a Rhodococcus rhodochrous strain that degrades 2-ethoxyphenol and 4-methoxybenzoate. J Bacteriol 175, 1467-1474.

Koga, Y., Nishihara, M., Morii, H. \& Akagawa-Matsuhita, M. (1993). Ether polar lipids of methanogeneic bacteria: structures, comparative aspects, and biosyntheses. Microbiol Rev 57, $164-182$.

Kroppenstedt, R. M., Möller, B. \& Hippe, H. (1985). Quinones and Cellular Fatty Acids in Anaerobic Bacteria. Abstracts of the ASM (German) local branch meeting, Würzburg, Germany.

Lane, D. J. (1991). $16 \mathrm{~S} / 23 \mathrm{~S}$ sequencing. In Nucleic Acid Techniques in Bacterial Systematics, pp. 115-175. Edited by E. Stackebrandt \& M. Goodfellow. Chichester: Wiley.

Lapage, S. P., Sneath, P. H. A., Lessel, E. F., Skerman, V. B. D., Seeliger, H. P. R. \& Clark, W. A. (editors) (1992). International Code of Nomenclature of Bacteria (1990 Revision). Bacteriological Code. Washington, DC: American Society for Microbiology.

Maidak, B. L., Cole, J. R., Parker, C. T., Jr \& 11 other authors (1999). A new version of the RDP (Ribosomal Database Project). Nucleic Acids Res 27, 171-173.

Mesbah, M., Premachandran, U. \& Whitman, W. B. (1989). Precise measurement of the $\mathrm{G}+\mathrm{C}$ content of deoxyribonucleic acid by high-performance liquid chromatography. Int J Syst Bacteriol 39, 159-167.

Miller, L. T. (1982). Single derivatization method for routine analysis of bacterial whole-cell fatty acid methyl esters, including hydroxy acids. $J$ Clin Microbiol 26, 584-586.

Miyagawa, E., Azuma, R. \& Suto, T. (1979). Cellular fatty acid composition in Gram-negative obligately anaerobic rods. J Gen Appl Microbiol 25, 41-51.

Möller, B., Oßmer, R., Howard, B. H., Gottschalk, G. \& Hippe, H. (1984). Sporomusa, a new genus of gram-negative anaerobic bacteria including Sporomusa sphaeroides spec. nov. and Sporomusa ovata spec. nov. Arch Microbiol 139, 388-396.

Moore, L. V. H., Bourne, D. M. \& Moore, W. E. C. (1994). Comparative distribution and taxonomic value of cellular fatty acids in thirty-three genera of anaerobic Gram-negative bacilli. Int $J$ Syst Bacteriol 44, 338-347.

Mullis, K. B. \& Faloona, F. (1987). Specific synthesis of DNA in vitro via a polymerase-catalyzed chain reaction. Methods Enzymol 155, 335-350.

Neefs, J.-M., van de Peer, Y., De Rijk, P., Gloris, A. \& De Wachter, R. (1991). Compilation of small ribosomal subunit RNA sequences. Nucleic Acids Res 19, 1987-2015.

Ouattara, A. S., Traore, A. S. \& Garcia, J.-L. (1992). Characterization of Anaerovibrio burkinabensis sp. nov., a lactatefermenting bacterium isolated from rice field soils. Int $J$ Syst Bacteriol 42, 390-397.

Patel, B. K. C., Love, C. A. \& Stackebrandt, E. (1992). Helix 6 of the 16S rRNA of the bacterium Desulfotomaculum australicum 
exhibits an unusual structural idiosyncrasy. Nucleic Acids Res 20, 5483.

van de Peer, Y., Nicolai, S., Rijk, P. D. \& Wachter, R. D. (1996). Database on the structure of small ribosomal subunit RNA. Nucleic Acids Res 24, 86-91.

Prins, R. A. (1986). Genus X. Anaerovibrio Hungate 1966, $80^{\mathrm{AL}}$. In Bergey's Manual of Systematic Bacteriology, vol. 1, pp. 653-655. Edited by P. H. A. Sneath, N. S. Mair, M. E. Sharpe \& J. G. Holt. Baltimore: Williams \& Wilkins.

Prins, R. A., Akkermans-Kryswijk, J., Franklin-Klein, W., Kankhorst, A. \& van Golde, L. M. G. (1974). Metabolism of serine and ethanolamine plasmalogens in Megasphaera elsdenii. Biochim Biophys Acta 348, 361-369.

Rainey, F. A., Ward-Rainey, N. L., Janssen, P. H., Hippe, H. \& Stackebrandt, E. (1996). Clostridium paradoxum DSM $7308^{\mathrm{T}}$ contains multiple 16S rRNA genes with heterogeneous intervening sequences. Microbiology 142, 2087-2095.

Reynolds, E. S. (1963). The use of lead citrate at high $\mathrm{pH}$ as an electron-opaque stain in electron microcopy. J Cell Biol 17, 208-212.

Sadek, F., Drucker, D. B., Boote, V., Bennet, K. W. \& Eley, A. (1998). Phospholipids of Fusobacterium spp. J Appl Microbiol 85, 302-308.

Schauder, R. \& Schink, B. (1989). Anaerovibrio glycerini sp. nov., an anaerobic bacterium fermenting glycerol to propionate, cell matter, and hydrogen. Arch Microbiol 152, 473-478.

Schleifer, K. H., Leuteritz, M., Weiss, N., Ludwig, W., Kirchhof, G. \& Seidel-Rüfer, H. (1990). Taxonomic study of anaerobic, Gramnegative, rod-shaped bacteria from breweries: emended description of Pectinatus cerevisiiphilus and description of Pectinatus frisingensis sp. nov., Selenomonas lacticifex sp. nov., Zymophilus raffinosivorans gen. nov., sp. nov., and Zymophilus paucivorans sp. nov. Int $J$ Syst Bacteriol 40, 19-27.

Shah, H. N., Collins, M. D. \& Kroppenstedt, R. M. (1983). Biochemical and chemical studies on Bacteroides multiacida and Bacteroides hypermegas. J Appl Bacteriol 55, 151-158.

Spurr, A. R. (1969). A low viscosity epoxy resin embedding medium for electron microscopy. J Ultrastruct Res 28, 31-43.
Stackebrandt, E., Pohla, H., Kroppenstedt, R., Hippe, H. \& Woese, C. R. (1985). 16S rRNA analysis of Sporomusa, Selenomonas, and Megasphaera: on the phylogenetic origin of Gram-positive eubacteria. Arch Microbiol 143, 270-276.

Stackebrandt, E., Sproer, C., Rainey, F. A., Burghardt, J., Päuker, O. \& Hippe, H. (1997). Phylogenetic analysis of the genus Desulfotomaculum: evidence for the misclassification of Desulfotomaculum guttoideumi and description of Desulfotomaculum orientis as Desulfosporosinus orientis gen. nov., comb. nov. Int $J$ Syst Bacteriol 47, 1134-1139.

Stahl, E. (1967). Dünnschichtchromatographie. Berlin: Springer.

Stankewich, J. P., Cosenza, B. J. \& Shigo, A. L. (1971). Clostridium quercicolum sp. n., isolated from discolored tissues in living oak trees. Antonie Leeuwenhoek 37, 299-302.

Stoesser, G., Tuli, M. A., Lopez, R. \& Sterk, P. (1999). The EMBL nucleotide sequence databases. Nucleic Acids Res 27, 17-24.

Tamaoka, J. \& Komagata, K. (1984). Determination of DNA base composition by reversed-phase high-performance liquid chromatography. FEMS Microbiology Lett 25, 125-128.

Tindall, B. J. (1990a). A comparative study of the lipid composition of Halobacterium saccharovorum from various sources. Syst Appl Microbiol 13, 128-130.

Tindall, B. J. (1990b). Lipid composition of Halobacterium lacusprofundi. FEMS Microbiol Lett 66, 199-202.

Tindall, B. J. (1996). Respiratory lipoquinones as biomarkers. In Molecular Microbial Ecology Manual, supplement 1, section 4.1.5, pp. 1-21. Edited by A. Akkermans, F. de Bruijn \& D. van Elsas. Dordrecht: Kluwer.

Verkley, A. J., Ververgaert, P. H. J. T., Prins, R. A. \& van Golde, L. M. G. (1975). Lipid-phase transitions of the strictly anaerobic bacteria Veillonella parvula and Anaerovibrio lipolytica. $J$ Bacteriol 124, 1522-1528.

Watanabe, T., Okuda, S.-I. \& Takahashi, H. (1982). Physiological importance of even-numbered fatty acids and aldehydes in plasmalogen phospholipids of Selenomonas ruminantium. J Gen Appl Microbiol 28, 23-33.

Woese, C. R. (1987). Bacterial evolution. Microbiol Rev 51, 221-271 